\title{
ECONOMÍA CREATIVA EN LAAGLOMERACIÓN METROPOLITANA DE SEVILLA: AGENTES, REDES LOCALES DE COLABORACIÓN Y PRINCIPALES ACTUACIONES*
}

\author{
Inmaculada Caravaca Barroso \\ Gema González Romero \\ Víctor Fernández Salinas \\ Departamento de Geografía Humana. Universidad de Sevilla \\ caravaca@us.es; gemagonzalez@us.es; salinas@us.es \\ Antonio García García \\ Departamento de Geografía, Historia y Filosofía, Universidad Pablo de Olavide \\ agargar1@upo.es
}

\section{RESUMEN}

Desde hace ya más de una década se viene atribuyendo un valor estratégico a la economía creativa al entender que puede contribuir a estimular la creación de empleo y a promover nuevos modelos de desarrollo territorial. Con este contexto general de referencia, el objetivo de este artículo es contribuir al conocimiento de la economía creativa en la aglomeración metropolitana de Sevilla, centrando especialmente la atención en los agentes a ella vinculados, en las redes de cooperación socio-institucional que entre dichos agentes se conforman y en sus principales actuaciones.

Palabras clave: Economía creativa, redes de cooperación socio-institucional, aglomeración metropolitana de Sevilla.

\section{ABSTRACT}

For over a decade a strategic value has been placed on the so-called creative economy in the understanding that it can help to drive both job creation and promote new econo-

Fecha de recepción: enero 2012.

Fecha de aceptación: febrero 2013.

* Este artículo se integra en el Proyecto de Investigación del Plan Nacional de I+D+i COS2009-10888. 
mic models. Given this general reference context, the aim of this article is to contribute to knowledge of the creative economy in the Urban Agglomeration of Seville, with special focus on the public and private agents with links to the area, on the local socio-institutional cooperation networks created by these agents, and on their main actions.

Key words: creative economy, socio-institutional cooperation networks, Urban Agglomeration of Seville.

\section{INTRODUCCIÓN}

Desde hace ya más de una década se viene atribuyendo un valor estratégico a la llamada economía creativa al considerar que puede contribuir a estimular la creación de empleo y a promover un nuevo modelo económico, así como el desarrollo de ciudades y territorios (Bayliss, 2007; Cooke y Lazzaretti, 2008; Pratt, 2008; Evans, 2009; Foord, 2009). Se entiende como tal la que incluye actividades directa o indirectamente ligadas a la cultura, como es el caso, por ejemplo, de las relacionadas con el teatro, el cine y la televisión; la edición de libros; la reproducción de música, el diseño y la fotografía.

Junto a lo anterior, no puede dejarse al margen que se insiste también en la importancia de la puesta en valor de los recursos, no sólo para propiciar el crecimiento económico, sino también el desarrollo de las sociedades y los territorios, generalizándose la tesis de que resulta fundamental la utilización imaginativa, racional, equilibrada y dinámica de todas las formas de capital y bienes patrimoniales, ya sean estos monetarios, humanos, naturales, culturales, sociales o territoriales (CEPAL, 1991 y 1992; Allende, 1995; Caravaca y González, 2009). Esto supone, por consiguiente, la revalorización de la cultura y del llamado capital cultural a ella asociado al considerarlo un importante recurso, además de un factor de cohesión social y de aprendizaje colectivo dada su capacidad para aportar respuestas creativas e imaginativas con las que solucionar problemas y disfunciones e impulsar procesos de desarrollo de carácter integrado (Rao, 1998; Ortega Valcárcel, 1998 y 2004).

Relacionando estas tesis puede entenderse el interés despertado por la economía creativa, puesto que el crecimiento de las actividades que la conforman está en buena parte sustentado en la existencia de recursos patrimoniales, entre los que hay que destacar algunos muy específicos, como el conocimiento y la creatividad, que son muy difíciles de deslocalizar, lo que los hace especialmente interesantes para promover procesos de desarrollo territorial (Rallet, 1995). No puede extrañar, en consecuencia, que se haya subrayado la importancia de aquellos recursos ligados a la propia identidad cultural y a la capacidad creativa al considerarlos posibles bases que sustenten nuevos modelos de desarrollo económicamente competitivos, ambientalmente sostenibles y territorialmente equilibrados (Mas Serra, 2009).

Un primer detonante de la creciente valoración de las actividades creativas fue el impulso institucional dado en Gran Bretaña a su desarrollo (Department of Culture, Media and Sports, 1998), mantenido después por otras instituciones internacionales (UNESCO, 2006 y 2008; UNCTAD, 2004 y 2008) y muy especialmente por la Unión Europea (2006), que las integra en la Estrategia Europa que surge de la Cumbre de Lisboa del año 2000 y que se actualizó después con propuestas cuyo horizonte llega al año 2020. Dentro de sus actuaciones, la Unión Europea, además de diseñar un Libro Verde cuyo objeto es liberar el potencial de estas 
actividades, declaró 2009 Año Europeo de la Creatividad y la Innovación (CE, 2009 y 2010 a y b). Hay que tener en cuenta, además, que el énfasis que la Unión Europea está poniendo sobre este tipo de actividades obedece, también, al reconocimiento de que éstas pueden contribuir al cambio económico estructural que permita la superación de la crisis (CE, 2010c).

No puede extrañar que, como contrapunto a estos discursos y propuestas, haya surgido también una corriente crítica que los cuestiona. Por una parte, se subraya una cierta imprecisión de la categoría conceptual de economía creativa y, por otra, la excesiva capacidad que se le está atribuyendo como promotora del desarrollo económico. En cuanto a la ambigüedad conceptual, ciertos autores intentan evidenciar las diferencias existentes entre las actividades creativas y la creatividad, entendiendo, desde la perspectiva económica, que esta última no sólo es propia de ciertas actividades sino que puede afectar al conjunto de la economía (Bouquillion y MiègeMoeglin, 2011). A su vez, la indefinición preocupa a algunas instituciones, por lo que dirigen sus esfuerzos al intento de identificar a los sectores que conforman a la economía creativa, como se pone de manifiesto en las clasificaciones utilizadas por UNESCO (2006: 3), UNCTAD, (2008: 14) y la Unión Europea (2010a: 6). Por otra parte, son varios los autores que cuestionan el excesivo énfasis que se está poniendo en la potencialidad socio-económica de este tipo de actividades al entender que están sirviendo para justificar intereses ideológicos, económicos y de poder muy ligados a la globalización de los mercados y a concepciones económicas neoliberales (Bustamante, ed., 2011); en este último sentido, sirve como ejemplo el discurso de Tremblay (2011) subrayando que con la promoción de estas actividades lo que se pretende es reforzar el reconocimiento de la propiedad intelectual y priorizar el pago de los derechos de autor.

Pero, con todos los matices y cautelas requeridos, desde una perspectiva territorial las actividades creativas parecen estar demostrando una cierta capacidad para generar nuevas oportunidades de desarrollo socioeconómico en determinados ámbitos; capacidad que, a priori, no debe despreciarse. La puesta en valor de ese recurso intangible que es la creatividad, en este caso asociada a la cultura, puede contribuir no sólo al mero crecimiento económico sino, y sobre todo, al desarrollo territorial, lo que depende en última instancia de las capacidades de los agentes para movilizar a la sociedad local con el fin de generar y atraer actividades que aúnen cultura y mercado. Esto último exige que para profundizar en el conocimiento de las actividades creativas en ciudades y territorios sea imprescindible analizar el comportamiento de aquellos agentes cuyas actuaciones incidan en ellas; y no hay que olvidar que dichas actuaciones están muy relacionadas con la capacidad de conformar redes de cooperación socio-institucional, estrechamente asociadas a la gobernanza.

En este contexto general de referencia, el objetivo de este artículo es contribuir al conocimiento de la economía creativa en la aglomeración metropolitana de Sevilla, centrando especialmente la atención en los agentes públicos y privados que en ella operan, en las redes socio-institucionales locales que entre ellos se conforman y en sus principales actuaciones.

Para intentar alcanzar estos objetivos, se parte de las siguientes preguntas de investigación: ¿Cuál es el peso de la economía creativa en la aglomeración metropolitana de Sevilla? ¿Cuántas y cuáles son las instituciones públicas que están incidiendo en el comportamiento de dichas actividades? ¿Qué otros organismos y agentes con presencia activa en el área están relacionados con ellas? ¿Se han creado redes de cooperación socio-institucional entre agentes públicos y privados con incidencia en las actividades creativas? ¿Quiénes participan en ellas? ¿Qué tipos de actuaciones y estrategias se están implementando? 
Desde el punto de vista metodológico, para la identificación de las actividades creativas se ha utilizado como referencia la propuesta realizada por Naciones Unidas (UNCTAD, 2008), adaptada en este caso a la información disponible en España en función de las agrupaciones de actividad de la Clasificación Nacional de Actividades Económicas (CNAE 2009) (Méndez, Michelini, Prada y Tébar, 2012, Fig. 1).

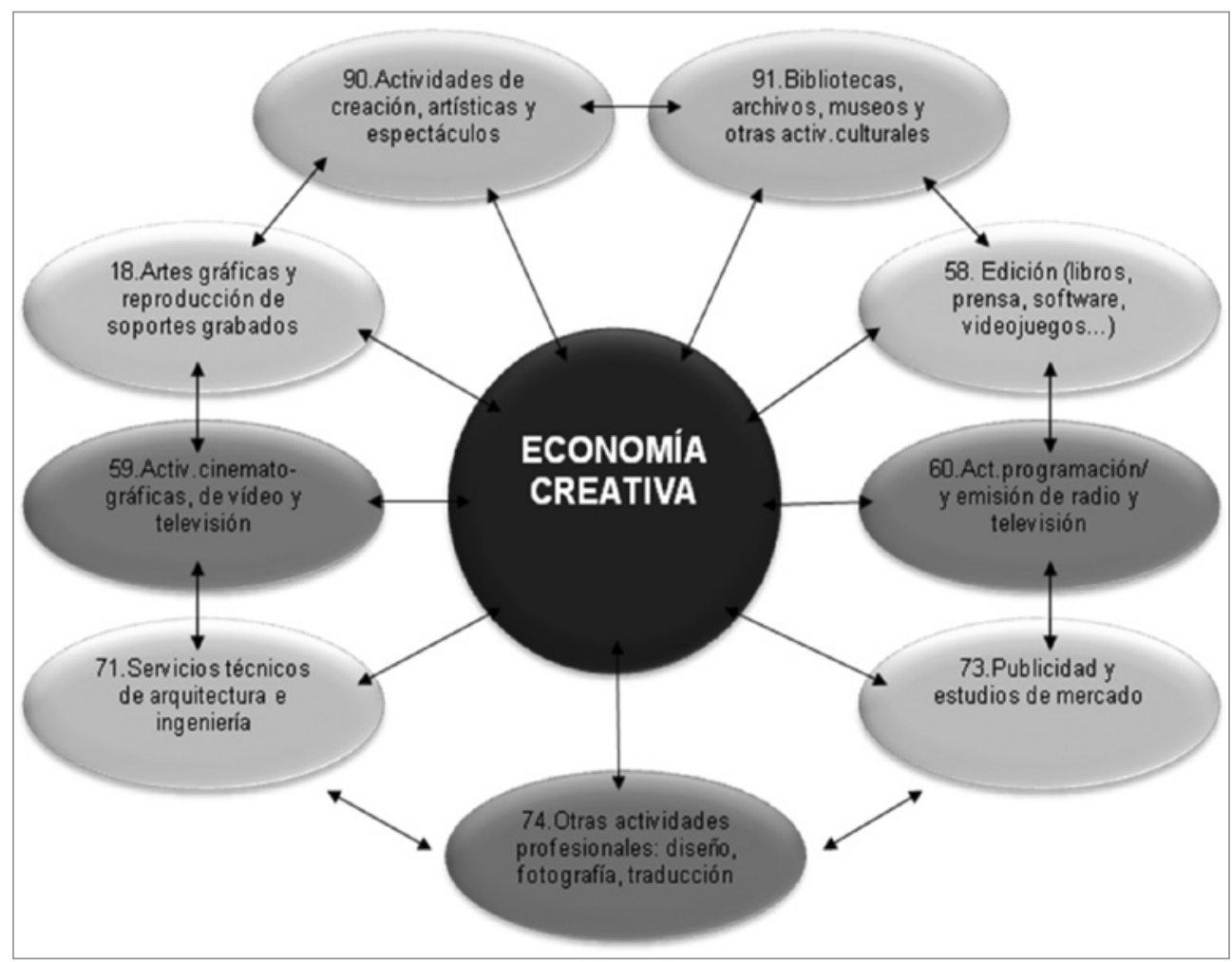

Fuente: Méndez, Michelin, Prada y Tébar, 2012: 12

Las fuentes utilizadas para llevar a cabo esta investigación son de tres tipos. Por una parte, las de carácter cuantitativo, que se centran en el registro de la Seguridad Social a 31 de diciembre de 2009 (Tesorería General de la Seguridad Social), con datos disponibles sobre número de empresas y de trabajadores ${ }^{1}$. Por otra parte, y teniendo en cuenta que la información cuantitativa no es suficiente para profundizar en el análisis de estas actividades, se ha utilizado otra infor-

1 Tal y como ya se señalaba en anteriores trabajos de investigación, es de lamentar que, debido al secreto estadístico, sólo se haya podido contar con información a dos dígitos de la Clasificación Nacional de Actividades Económicas (CNAE), lo que explica que se incluyan con las actividades creativas algunas otras que no lo son, como es el caso de las de ingeniería y de estudios de mercado. 
mación complementaria de carácter cualitativo que permite conocer la forma en que actúan los diferentes actores implicados y que ha resultado de una gran utilidad para comprender algunas de las lógicas subyacentes. Dicha información se ha generado a partir de la realización de 37 entrevistas semi-estructuradas de aproximadamente dos horas de duración que, aunque partiendo de un guión inicial, han permitido a los entrevistados expresarse con entera libertad sobre todos aquellos aspectos que han considerado de interés. Dichas entrevistas se realizaron a informantes clave procedentes de diversas instituciones, organizaciones, fundaciones, asociaciones y empresas durante los años 2010 y 2011. Para la selección de los entrevistados se han considerado tres criterios: los tipos de agentes implicados, su pertenencia a los municipios con mayor presencia de actividades creativas y su vinculación a los sectores que generan más empleo en el área; se han priorizado, además, aquellos agentes que han sido referenciados por otros en las entrevistas. Se asume así, una vez más, que para lograr los objetivos propuestos resulta imprescindible utilizar metodologías de carácter cualitativo que permitan profundizar en el conocimiento de la realidad territorial objeto de atención. Por último, se han consultado documentos elaborados por las distintas instituciones con presencia activa en el área, así como las páginas webs de agentes públicos y privados.

El artículo se estructura en seis epígrafes. Tras una presentación introductoria, se incluye un segundo apartado en el que se analiza la presencia de las actividades creativas en la aglomeración metropolitana de Sevilla, considerando su peso en el conjunto de Andalucía y diferenciando también entre lo que ocurre en Sevilla capital y en su corona metropolitana. En el apartado tercero y cuarto se consideran las estrategias y actuaciones llevadas a cabo por los principales agentes, tanto públicos como privados, ya se traten de administraciones, organismos, asociaciones o empresas. El epígrafe quinto, se dedica a analizar la red de cooperación socio-institucional que entre los mismo se conforma. Se cierra el trabajo con las conclusiones pertinentes.

\section{LAS ACTIVIDADES CREATIVAS EN LA AGLOMERACIÓN METROPOLITANA DE SEVILLA}

La aglomeración metropolitana de Sevilla ocupa una superficie de $4.900 \mathrm{Km}^{2}$ e incluye una población de 1.508.605 habitantes (Padrón Municipal de Habitantes, 2010). Las debilidades estructurales y las malas prácticas coyunturales que han venido obstaculizando el proceso de desarrollo en la Comunidad Autónoma de Andalucía han afectado también a la aglomeración, haciendo necesario un gran esfuerzo de las empresas, de las instituciones y de la sociedad en su conjunto para superar los problemas derivados de tal circunstancia. En estrecha relación con lo anterior, no cabe ninguna duda de que resulta imprescindible la puesta en valor de todos los recursos disponibles, y muy especialmente de aquellos vinculados a la creatividad que, como ya se ha mencionado, se sustentan en parte en manifestaciones culturales de fuerte presencia en el área.

Centrando la atención en las actividades clasificadas como creativas, en la aglomeración metropolitana de Sevilla en 2009 se registraban 2.165 empresas que daban empleo a 28.657 trabajadores, representando tan sólo un 4,81\% y un 5,17\%, respectivamente, de la economía del área. No obstante, no puede dejarse al margen la importante concentración regional de estas actividades en la aglomeración, puesto que suponen un 25,99\% de las empresas y un 36,35\% de los empleos existentes en Andalucía; cifras que superan sobradamente a los correspondientes al conjunto de las actividades económicas (13,13\% y 19,58\%; Cuadro 1). Dicha concentra- 
ción se produce también y mucho más intensamente dentro de la aglomeración, puesto que en la ciudad central se localiza un 67,25\% de las empresas y un 67,32\% de los empleos.

De entre estas actividades destacan sobre todo las de Arquitectura e ingeniería que, con 883 empresas y 11.676 trabajadores, suponen un $26,62 \%$ y 39,58\% de los existentes en Andalucía; estos sectores son seguidos a bastante distancia por las de Publicidad y estudios de mercado, cuyas 358 empresas dan empleo a 4.370 trabajadores $(29,22 \%$ y $37,99 \%$, respectivamente, de la región). Se trata en ambos casos de sectores que integran actividades a las que puede cuestionarse su relación con la cultura, como es el caso de las de Ingeniería y de los Estudios de mercado. Resultan también reseñables, dado el empleo que generan, las ramas de Diseño y fotografía, con 201 empresas y 2.817 trabajadores (22,02\% y 30,44\% de Andalucía); Cine, televisión y música, con 136 empresas y 2.608 trabajadores (33,01\% y 48,15\%); y Creación artística y espectáculos, cuyos empresas suman 131 y dan empleo a 2.330 trabajadores $(28,73 \%$ y $37,53 \%$ ). Ninguna de las restantes ramas creativas logra superar los 2.000 empleos (Cuadro 1).

Cuadro 1

NÚMERO DE EMPRESAS Y TRABAJADORES EN ACTIVIDADES CREATIVAS (2009)

\begin{tabular}{|c|c|c|c|c|c|c|c|c|}
\hline & \multicolumn{2}{|c|}{$\begin{array}{c}\text { Total } \\
\text { Aglomeración }\end{array}$} & \multicolumn{2}{|c|}{ Sevilla } & \multicolumn{2}{|c|}{$\begin{array}{c}\text { Corona } \\
\text { Metropolitana } \\
\end{array}$} & \multicolumn{2}{|c|}{$\begin{array}{l}\text { Aglomeración/ } \\
\text { Andalucía (\%) }\end{array}$} \\
\hline & Emp. & Trabaj. & Emp. & Trabaj. & Emp. & Trabaj. & Emp. & Trabaj. \\
\hline $\begin{array}{l}\text { Artes gráficas y } \\
\text { reproducción de } \\
\text { soportes grabados }\end{array}$ & 225 & 1.908 & 121 & 976 & 104 & 932 & 20,83 & 26,68 \\
\hline Edición & 148 & 1.860 & 111 & 1.292 & 37 & 568 & 28,35 & 32,66 \\
\hline $\begin{array}{l}\text { Cine, televisión, } \\
\text { música, etc. }\end{array}$ & 136 & 2.608 & 72 & 861 & 64 & 1.747 & 33,01 & 48,15 \\
\hline $\begin{array}{l}\text { Programación y } \\
\text { emisión de radio } \\
\text { y TV }\end{array}$ & 41 & 657 & 22 & 498 & 19 & 159 & 17,67 & 28,37 \\
\hline $\begin{array}{l}\text { Arquitectura e } \\
\text { ingeniería }\end{array}$ & 883 & 11.678 & 629 & 9.057 & 254 & 2621 & 26,62 & 39,58 \\
\hline $\begin{array}{l}\text { Publicidad y } \\
\text { estudios de mercado }\end{array}$ & 358 & 4.370 & 251 & 2.810 & 107 & 1.560 & 29,22 & 37,99 \\
\hline $\begin{array}{l}\text { Diseño, fotografía, } \\
\text { etc. }\end{array}$ & 201 & 2.817 & 128 & 1.683 & 73 & 1.134 & 22,02 & 30,44 \\
\hline $\begin{array}{l}\text { Creación artística y } \\
\text { espectáculos }\end{array}$ & 131 & 2.330 & 90 & 1.743 & 41 & 587 & 28,73 & 37,53 \\
\hline $\begin{array}{l}\text { Bibliotecas, } \\
\text { archivos y museos }\end{array}$ & 42 & 429 & 32 & 372 & 10 & 57 & 24,42 & 24,09 \\
\hline $\begin{array}{l}\text { TOTAL } \\
\text { ECONOMÍA } \\
\text { CREATIVA }\end{array}$ & 2.165 & 28.657 & 1.456 & 19.292 & 709 & 9.365 & 25,99 & 36,35 \\
\hline $\begin{array}{l}\text { Resto actividades } \\
\text { económicas }\end{array}$ & 42.891 & 525.397 & 27.204 & 311.779 & 15.687 & 21.3618 & 12,81 & 19,10 \\
\hline $\begin{array}{l}\text { TOTAL } \\
\text { ECONOMÍA }\end{array}$ & 45.056 & 554.054 & 28.660 & 331.071 & 16.396 & 222.983 & 13,13 & 19,58 \\
\hline
\end{tabular}

Fuente: Tesorería General de la Seguridad Social. Elaboración propia. 
Como complemento del cuadro 1 se han realizado diagramas estrellas que permiten matizar el análisis de la distribución sectorial y territorial de las actividades creativas (Fig. 2) ${ }^{2}$. Tal y como se observa en las figuras 2 a y 2 b, la distribución sectorial en la aglomeración metropolitana y en el resto de Andalucía presenta ciertas diferencias, aunque en ambos casos destaca la rama de Publicidad y Estudios de mercado. Más acentuadas son las diferencias existentes en el seno de la aglomeración (Fig. 2c y 2d). Así, la ciudad central: el municipio de Sevilla, está más especializada en la industria Editorial y las Actividades de creación artística, lo que habría que relacionar con su condición de capital regional y el hecho de ser la mayor población de Andalucía. Si las editoriales buscan la cercanía a los principales organismos e instituciones educativas y culturales, las Actividades de creación artística persiguen, además, la mayor concentración de oferta y demanda cultural. A diferencia de lo anterior, la corona metropolitana está mucho más especializada que el municipio central en Cine, Televisión y Música, así como en Artes Gráficas y Reproducción de Soportes Grabados; en este caso, la localización de los estudios de producción y emisión de la televisión pública andaluza en la zona oeste de la corona pueden haber motivado la concentración de estas actividades más allá de la ciudad central.

Figura 2

ESTRUCTURA SECTORIAL DE LAS ACTIVIDADES CREATIVAS SEGÚN NÚMERO DE TRABAJADORES (2009)

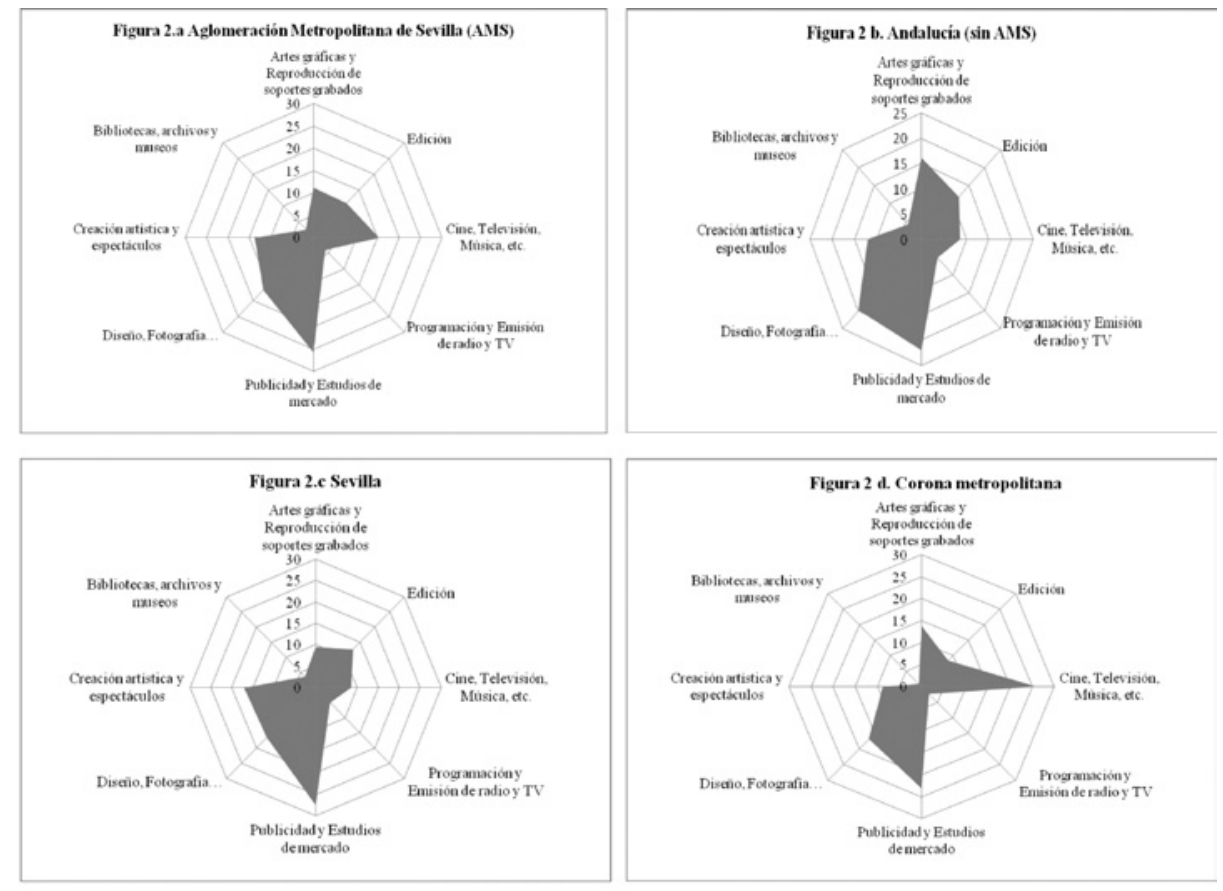

Fuente: Tesorería General de la Seguridad Social y elaboración propia.

2 La rama Arquitectura e Ingeniería (actividades que las fuentes no permiten diferenciar entre sí) no se ha incluido en los diagramas para evitar que su alto peso relativo en el total impida observar con nitidez la distribución sectorial de las actividades creativas. 
La existencia de externalidades positivas de muy distinto signo ayudan a entender la tendencia concentradora de estas actividades, aunque resulta obvio que en la escala local la tradición histórica y cultural, el patrimonio material e inmaterial disponible, además de las políticas implementadas pueden resultar determinantes en el crecimiento de este tipo de actividades.

Siguiendo esta lógica general, en el conjunto de Andalucía la aglomeración metropolitana de Sevilla es el foco de mayor concentración de la economía creativa. No obstante, no puede dejarse al margen que el peso del empleo generado por estas actividades en el conjunto de la economía sigue siendo muy limitado $(4,81 \%)$, aunque existan ligeras diferencias en el seno de la aglomeración entre municipio central $(5,8 \%)$ y el resto del área $(4,2 \%)$. Tal y como se señalaba anteriormente, la concentración de estas actividades dentro de la aglomeración es más que significativa dado que Sevilla reúne el 67,25\% de las empresas y el 67,32\% de los empleos. «La economía creativa contribuye, por tanto, de forma bastante modesta a las tendencias difusoras hoy habituales que favorecen la configuración de metrópolis policéntricas y de carácter difuso» (Méndez, Michelini, Prada y Tébar, 2012: 22).

\section{ACTUACIONES REALIZADAS POR LOS AGENTES PÚBLICOS}

Durante las últimas décadas y desde distintas disciplinas sociales, se han llevado a cabo numerosas reflexiones y análisis sobre el papel que ejercen los agentes socio-institucionales en los procesos de desarrollo territorial. Se subraya en estos trabajos que la capacidad que muestran dichos agentes para aprovechar los propios recursos -convirtiendo los que son genéricos en específicos- resulta esencial para dinamizar el entramado socioeconómico de los territorios, reforzando así sus ventajas competitivas y logrando, con ello, su mejor inserción en el espacio global de las redes. Hay que tener en cuenta, además, que el papel que desempeña cada uno de estos agentes está condicionado por las normas y reglas de actuación propias de los distintos ámbitos a los que pertenecen, por las estructuras económicas heredadas y por los recursos con que éstos cuentan.

Para poder profundizar en el conocimiento de la economía creativa resulta imprescindible analizar la forma en la que operan las instituciones públicas con incidencia en el área a través de las estrategias y actuaciones que vienen desarrollando. Para ello, se ha contado, por una parte, con información procedente de la documentación generada por dichas instituciones y, por otra, con las entrevistas en profundidad que se les han realizado y que han servido de una gran ayuda al permitir matizar la información documental.

Tal y como muestra el cuadro 2, que intenta sintetizar los distintos tipos de actuaciones realizadas, son diversos los campos en los que cada uno de estos actores está trabajando. Según el número de campos en los que actúan destacan, por una parte, el Proyecto Lunar, desarrollado por las consejerías de Innovación, Ciencia y Empresa y de Empleo de la Junta de Andalucía. No obstante, hay que tener en cuenta que, más que el número de campos en los que los agentes entrevistados desarrollan actividades, interesa sobre todo conocer el grado de solidez de las actuaciones y, en este sentido, junto al organismo citado, hay que hacer referencia a otros dos, dependientes en este caso del Ayuntamiento de Sevilla: Sevilla Global ${ }^{3}$ y el Centro de Recursos Empresariales Avanzados, cuyas intervenciones, pese a estar realizadas a escala local, han

3 Esta agencia de desarrollo ha sido desmantelada recientemente. 
sido bastante significativas. Junto al Ayuntamiento de Sevilla hay que mencionar también al de Alcalá de Guadaíra, que viene desarrollando en los últimos años un papel muy activo en el impulso de la innovación empresarial y, recientemente, de las actividades creativo-culturales.

Por otra parte, respecto a la mayor o menor presencia de las actividades incluidas en unos u otros campos, es en el de Formación en el que se generan más actuaciones. Ocupan un segundo lugar en esta jerarquía las actuaciones realizadas para impulsar la creación de Espacios y equipamientos empresariales, así como las que se incluyen en el campo de Otras actividades y Organización de Espectáculos. Son también bastantes los agentes involucrados en el Asesoramiento Técnico, los que realizan tareas relacionadas con la $I+D+i$ y los que llevan a cabo Análisis, diagnósticos o planificación; mientras son menos lo que cuentan con presupuestos para incentivar a las empresas. Esto último resulta bastante ilustrativo de los cambios experimentados respecto a la forma de concebir la promoción de actividades empresariales, antes muy centradas en la concesión de ayudas y subvenciones, y ahora fundamentalmente dirigidas a la prestación de servicios.

Cuadro 2

ACTUACIONES DE LAS ADMINISTRACIONES Y ORGANISMOS PÚBLICOS VINCULADOS A LAS ACTIVIDADES CREATIVAS

\begin{tabular}{|c|c|c|c|c|c|c|c|c|c|}
\hline \multicolumn{2}{|l|}{ Entrevistado } & $\begin{array}{c}\text { Análisis/ } \\
\text { Diagnóstico/ } \\
\text { Planificación }\end{array}$ & $\mathrm{I}+\mathrm{D}+\mathrm{i}$ & $\begin{array}{l}\text { Asesora- } \\
\text { miento } \\
\text { técnico }\end{array}$ & Formación & $\begin{array}{l}\text { Espacios/ } \\
\text { equipa- } \\
\text { mientos a } \\
\text { empresa }\end{array}$ & $\begin{array}{l}\text { Incen- } \\
\text { tivos }\end{array}$ & $\begin{array}{l}\text { Organización } \\
\text { espectáculos, } \\
\text { editorial... }\end{array}$ & $\begin{array}{c}\text { Otras } \\
\text { activida- } \\
\text { des }\end{array}$ \\
\hline UE & $\begin{array}{l}\text { EURO- } \\
\text { CEI }\end{array}$ & & & & & & & & \\
\hline \multirow{3}{*}{$\begin{array}{l}\text { Comunidad } \\
\text { Autónoma }\end{array}$} & IAPH & & & & & & & & \\
\hline & AAIC & & & & & & & & \\
\hline & PL & & & & & & & & \\
\hline $\begin{array}{l}\text { Diputación } \\
\text { Provincial }\end{array}$ & $\mathrm{ACPC}$ & & & & & & & & \\
\hline \multirow{3}{*}{$\begin{array}{l}\text { Ayuntamiento } \\
\text { Sevilla }\end{array}$} & CREA & & & & & & & & \\
\hline & SG & & & & & & & & \\
\hline & OAAS & & & & & & & & \\
\hline $\begin{array}{l}\text { Ayuntamiento } \\
\text { Alcalá de } \\
\text { Guadaíra }\end{array}$ & $\begin{array}{l}\text { Complejo } \\
\text { IDEAL }\end{array}$ & & & & & & & & \\
\hline \multirow{2}{*}{ Universidad } & CICUS & & & & & & & & \\
\hline & UPSC & & & & & & & & \\
\hline
\end{tabular}

EUROCEI: Centro Europeo de Empresas e Innovación; IAPH: Instituto Andaluz del Patrimonio Histórico; AAIC: Agencia Andaluza de Instituciones Culturales; PL: Proyecto Lunar; ACPC: Área de Ciudadanía, Participación y Cultura; CREA: Centro Recursos Empresariales Avanzados; SG: Sevilla Global; OAAS: Oficina Artesanía del Ayuntamiento de Sevilla; CICUS: Centro Iniciativas Culturales de la Universidad de Sevilla; UPSC: Unidad de Promoción Social y Cultural de la Universidad Pablo de Olavide.

Fuente: Elaboración propia a partir de las entrevistas y de la documentación disponible. 
Más allá de este análisis sintético, interesa especialmente observar la forma en que las instituciones públicas conciben las actividades creativas y cómo se implementan las actuaciones a ellas vinculadas. En general, la referencia a la importancia de las actividades creativas está presente en buena parte de los programas, proyectos y actuaciones realizados por las instituciones públicas de distintos niveles administrativos que operan en la aglomeración metropolitana de Sevilla.

En cuanto a los proyectos financiados por la Unión Europea a través de EUROCEI, los resultados del llamado Proyecto CISNET (Creative Industries Support Network), desarrollado en cooperación con otras regiones europeas, no han sido hasta ahora relevantes, pese al indudable interés de sus objetivos y planteamientos. Se trata de un proyecto dirigido a impulsar la competitividad de las PYMEs pertenecientes al sector de las industrias creativas a partir del apoyo al desarrollo de nuevos productos; el acceso a nuevos mercados; la adopción de mejoras técnicas; el fomento de la innovación; el establecimiento de una plataforma de comercio común y la colaboración interempresarial.

A nivel nacional, el Ministerio de Cultura diseñó en 2009 los Planes de Fomento de las Industrias Culturales y Creativas en cuyo título queda explícito su objetivo. A ello contribuye, además, que dentro del Plan Estadístico Nacional 2009-2011 se incluya la llamada Cuenta Satélite de la Cultura.

Dentro del marco establecido por el Plan de Innovación y Modernización de Andalucía (2006), el gobierno de esta comunidad autónoma hacía hincapié en la necesidad de potenciar el desarrollo de las empresas creativas. Para ello, se aprobó el denominado Proyecto Lunar, en el que se involucran las Consejerías de Empleo, e Innovación, Ciencia y Empresa. El objetivo de este proyecto es fortalecer la contribución de estas empresas a la economía andaluza, para lo que presta diferentes servicios: asesoramiento a emprendedores en la elaboración de planes de negocio, en la tramitación burocrática o en la búsqueda de vías de financiación; posibilidad de contar con consultorías especializadas; apoyo a la internacionalización y organización de jornadas formativas. En Sevilla, que fue la ciudad en la que se inició este proyecto, ya a fines de 2008 se habían adherido al mismo 346 proyectos empresariales, realizado 43 encuentros interempresariales y acciones de promoción, constituido 44 nuevas empresas y generado 61 empleos directos. Como complemento a lo anterior, se creó una incubadora especializada denominada La Fábrica de Ideas (Sánchez y Álvaro, 2009). Es reseñable que hayan sido varias las empresas entrevistadas que valoran muy positivamente las actuaciones asociadas a este proyecto, aunque, como contrapunto de lo anterior, el cambio de localización de su sede sevillana, que había sido elegido precisamente por su emplazamiento en una zona en la que se concentran actividades creativas, puede resultar una cortapisa que, unida a la crisis, frene su posible desarrollo.

Menos innovadoras parecen estar siendo, sin embargo, las actuaciones de la Consejería de Cultura a través de la Agencia Andaluza de Instituciones Culturales, que se concretan, sobre todo, en la concesión de ayudas para la programación de espectáculos y para la consolidación de espacios escénicos. Por su parte, el Instituto Andaluz de Patrimonio Histórico, también dependiente de la Consejería de Cultura, realiza tareas de formación y orientación profesional específicamente dirigidas a este tipo de actividades.

La Diputación Provincial, no está implicada directamente en potenciar las actividades creativas, y las tareas de fomento cultural que está desarrollando se sustentan en plantea- 
mientos muy tradicionales y dirigidos principalmente a la difusión. Dichas actividades se concretan en dos programas base: Fomento y Cooperación Cultural con los Municipios, y Circuito Provincial de Artes Escénicas y Municipales. El primero, que está dirigido fundamentalmente a los municipios de menor tamaño y, por tanto, con menos recursos para la cultura, se materializa en la realización de talleres, exposiciones y conciertos. El segundo se implementa en aquellos otros municipios que tienen más población, cuentan con teatros de cierta envergadura y, además, se comprometen a colaborar en la financiación de las actividades. Como antes se señalaba, es sólo de forma indirecta como esta institución incide en el posible desarrollo económico de las actividades creativas; hay que añadir a lo anterior el hecho de que, al igual que ocurre en otras instituciones, están disminuyendo considerablemente los presupuestos dedicados a tales fines y, en consecuencia, el número y la importancia de los proyectos financiados.

Centrando el análisis en la escala local, el Ayuntamiento de Sevilla ha realizado diversas actuaciones durante la última década. En 2003 aprobó el Plan Estratégico de la Cultura, que apostaba por la creatividad como base del proceso de desarrollo integral de la ciudad; en consecuencia, uno de los objetivos era potenciar el crecimiento de las empresas creativas para convertir Sevilla en lo que se denominaba Factoría Cultural. Junto a lo anterior, en 2005 se asumen los objetivos y líneas de actuación de la llamada Agenda 21 de la Cultura, con el compromiso de promover las actividades creativas y culturales. Como complemento de lo anterior, se crea un año más tarde el Instituto de la Cultura y las Artes, cuyo fin es consolidar la cultura y las actividades creativas de ella derivadas como uno de los principales motores de desarrollo urbano. Entre sus actuaciones, puede destacarse, por ejemplo, el fomento de la música, de lo que constituye un ejemplo especialmente significativo su apoyo a la Fundación Baremboin-Said, creada por la Junta de Andalucía en 2004 y con sede en Sevilla; uno de cuyos proyectos más significativos es la Orquesta West-Eastern Divan, formada por jóvenes músicos palestinos e israelíes. Estas últimas actuaciones están, además, estrechamente relacionadas con el reconocimiento de Sevilla en 2006 como Ciudad de la Música por parte de UNESCO, pasando con ello a formar parte de la Red de Ciudades Creativas de la Alianza Global para la Diversidad Cultural. Pese a la indudable importancia de tal nombramiento, llama bastante la atención la falta de información a la ciudadanía, hasta el punto de que muy generalizadamente desconoce este nombramiento.

Este Ayuntamiento creó, además, la agencia de desarrollo integral Sevilla Global, cuyo objetivo básico era implementar proyectos que fomentaran la economía y la creación de empleo. Se incluye, entre ellos, el de Creative Industries in Tradicional Intercultural Spaces que, financiado por la Unión Europea, tenía por objetivo la conformación de una red de ciudades interesadas en la promoción de las industrias creativas y culturales. Otro de los proyectos vinculado a las actividades creativas y también financiado por la Unión Europea es el Urban Network for Innovation in Ceramic, que integra a Sevilla con a otras ocho ciudades europeas de tradición ceramista, y que consolidará el reconocimiento a estas actividades tradicionales, especialmente tras la próxima inauguración del Museo de la Cerámica de Triana. Dentro de las actuaciones de Sevilla Global hay que destacar la creación del Parque Empresarial de Arte Sacro y Afines, estrechamente relacionado con un evento cultural de tanta importancia en la ciudad como la Semana Santa; aunque, lamentablemente, se trata de un proyecto fallido que no ha llegado a cumplir con los objetivos propuestos. Otra de las 
actuaciones de esta agencia de desarrollo es el Centro de Recursos Empresariales Avanzados (CREA) que, aunque funciona como una incubadora de empresas, también desarrolla proyectos; es el caso del titulado Interreg Cities, que a su vez tiene carácter europeo, siendo su objetivo analizar qué tipo de incubación resulta necesaria para las empresas vinculadas a la creatividad y a la cultura en su ámbito territorial de referencia. Tal y como se señaló anteriormente, el cambio de signo político del gobierno municipal, unido a la crisis, parecen ser las causas que han provocado el reciente desmantelamiento de esta agencia de desarrollo.

El Ayuntamiento de Sevilla cuenta, por último, con una oficina dedicada a prestar servicios a las empresas artesanas, para lo que se ha puesto en marcha el Programa Aprendices y se organizan diferentes mercados de artesanía.

De entre los ayuntamientos de la corona metropolitana que cuentan con una cierta presencia de las actividades creativas en sus estructuras productivas, hay que destacar el de Alcalá de Guadaíra, que en los últimos años se ha mostrado muy dinámico en el fomento de la innovación empresarial y de este tipo de actividades. Prueba de ello son los diferentes programas de servicios y asesoramiento empresarial, así como la creación de equipamientos e infraestructuras para propiciar el conocimiento y la innovación, entre los que destaca el Complejo de Innovación y Desarrollo de Alcalá de Guadaíra, que alberga al Centro de Apoyo a la Calidad y Seguridad, y al Centro de Apoyo al Diseño. A estos equipamientos se ha sumado recientemente el proyecto de una incubadora dirigida sólo a empresas del sector audiovisual.

A tenor de la información existente, el resto de los ayuntamientos de la aglomeración no parece estar prestando demasiada atención a las actividades clasificadas como creativas, por lo que no se ha considerado pertinente realizarles entrevistas. Aun así, cabría destacar a los de Dos Hermanas y Mairena del Aljarafe. El primero, lleva años desarrollando una política de creación de infraestructuras y equipamientos innovadores que se ha concretado en el Centro Tecnológico TIXE y el proyecto del Parque de Investigación y Desarrollo Dehesa de Valme. Por su parte, Mairena del Aljarafe cuenta con el Programa de apoyo empresarial IMPULSA del que se han beneficiado algunas empresas clasificadas como creativas.

Las dos universidades públicas existentes en el ámbito de estudio cuentan con varios programas y actuaciones para el fomento de las actividades creativas. La Universidad de Sevilla creó en 2008 el Centro de Iniciativas Culturales (CICUS), impulsando actividades de música, danza, teatro, cine, y organizando festivales, exposiciones, talleres y concursos en diferentes especialidades. Entre sus proyectos llama la atención el Laboratorio de Arte y Cultura Digital CicusLab, que tiene como principal objetivo la aplicación de las nuevas tecnologías digitales a las artes y a la cultura; a su vez, cabe destacar los premios concedidos a iniciativas empresariales innovadoras, entre las que han sido galardonadas empresas de sectores creativos. La otra universidad pública de Sevilla, la Pablo de Olavide, tiene un servicio de Extensión Cultural a partir del cual organiza diferentes eventos culturales y talleres de formación artística. Ambas universidades participan en el Observatorio Universitario Andaluz de la Cultura que, en colaboración con el resto de las universidades andaluzas y la Consejería de Economía, Innovación y Ciencia, han puesto en marcha proyectos colectivos de investigación, cursos de formación y organizado diferentes eventos relacionados con las actividades creativas.

Resulta paradójica la falta de coordinación entre instituciones que se evidencia en las duplicidades de actuaciones que contribuyen a su solapamiento. Esta situación es especialmente negativa en una situación de crisis como la actual en la que los fondos públicos son cada vez más exiguos. 


\section{CARACTERIZACIÓN DE LOS AGENTES PRIVADOS ENTREVISTADOS}

Junto a los agentes de carácter público, operan otros de carácter privado, tanto individuales como colectivos, que requieren también atención y de los que han sido entrevistados algunos de los más significativos.

La Fundación Cajasol tuvo en etapas anteriores una presencia muy activa en la organización de eventos culturales, prestando así un decidido apoyo a empresas y trabajadores autónomos ligados a la economía creativa. No obstante, como consecuencia del proceso de reestructuración a que se ha visto sometida la entidad y la consiguiente reorientación en su política, ha sufrido una reducción considerable del presupuesto para obra social y cultural, lo que ha supuesto la práctica supresión de la financiación de este tipo de actividades. .

Por su parte, han sido tres las federaciones y asociaciones empresariales entrevistadas. La Federación de Artesanos de Sevilla, que se escinde en 2009 de la de Andalucía, está compuesta por nueve asociaciones. Aunque su objetivo principal es promover el desarrollo de las empresas asociadas, se plantea también el reto de lograr que desaparezca la economía sumergida, hasta ahora con una alta presencia en el sector. Sus dos actuaciones principales se centran en colaborar estrechamente con el ayuntamiento en la organización de dos mercados artesanales, uno en primavera y otro en Navidad, en los que sólo participan artesanos con un cierto prestigio; a ellos hay que añadir la organización de la exposición denominada Pieza Única, dedicada a obras de diseño exclusivo, en la que colabora con la Junta de Andalucía para que las empresas obtengan la acreditación que supone la llamada Carta de Artesano.

La Asociación de Empresarios de Tecnologías de la Información y Comunicación (ETICOM), con sede social en Sevilla, se crea en 1998 para dar apoyo a las empresas andaluzas del sector y, entre los subsectores a los que sirve, se incluyen algunos de los considerados creativos: multimedia, infografía, industrias de contenidos... Sus actividades se centran en la prestación de todo tipo de servicios a las empresas asociadas (formación, proyección internacional, I+D, acceso a información tecnológica avanzada, celebración de encuentros...), contando, además, con instalaciones para computación en la nube, hemeroteca digital y centro de demostración. Sus centros de negocios se localizan no sólo en Andalucía sino en todo el territorio nacional así como en otros países entre los que se incluyen, por ejemplo, Polonia y China.

Por último, el Cluster Digital y Audiovisual de Andalucía (CLAND) es también una asociación de empresas sin ánimo de lucro de muy reciente creación (2010). Su finalidad es establecer cooperación y flujos de conocimiento entre las empresas asociadas para así hacer más competitivo el sector digital y audiovisual de la región. Lo conforman empresas muy diferentes tanto por su tamaño como por la actividad que desarrollan: videojuegos, plataformas web, aplicaciones móviles... CLAND está integrada en la Red Europa Intercluster, habiendo colaborado con ella muy recientemente para la organización del Creative and Digital Content Industrias Forum, celebrado en Sevilla en 2011.

Según puede observarse en el cuadro 3, es ETICOM la asociación que más tipos de servicios presta, seguida muy de cerca por CLAND y la Federación de Artesanos. Tal y como antes se comentaba, es de reseñar negativamente la considerable reducción de las actuaciones de un agente tan central en la ciudad de Sevilla como la Fundación Cajasol. 
Cuadro 3

ACTUACIONES DE LAS FUNDACIONES Y ASOCIACIONES EMPRESARIALES VINCULADAS A LAS ACTIVIDADES CREATIVO-CULTURALES

\begin{tabular}{|l|l|l|l|l|l|l|l|l|l|}
\hline Entrevistado & $\begin{array}{c}\text { Análisis/ } \\
\text { Diagnóstico/ } \\
\text { Planificación }\end{array}$ & I+D+i & $\begin{array}{c}\text { Asesora- } \\
\text { miento } \\
\text { técnico }\end{array}$ & Formación & $\begin{array}{c}\text { Espacios/ } \\
\text { equipa- } \\
\text { mientos a } \\
\text { empresa }\end{array}$ & Incentivos & $\begin{array}{c}\text { Organización } \\
\text { espectáculos, } \\
\text { editorial... }\end{array}$ & $\begin{array}{c}\text { Otras } \\
\text { activida- } \\
\text { des }\end{array}$ \\
\hline Fundación & FCS & & & & & & & & \\
\hline \multirow{2}{*}{$\begin{array}{l}\text { Asociación } \\
\text { de Empresas }\end{array}$} & ETICOM & & & & & & & & \\
\cline { 2 - 4 } & & & & & & & & \\
\hline
\end{tabular}

FCS: Fundación Cajasol; ETICOM: Asociación Andaluza de Empresas de Tecnologías de la Información y la Comunicación; FAS: Federación Artesanos Sevilla; CLAND: Cluster Digital y Audiovisual de Andalucía.

Fuente: Elaboración propia a partir de las entrevistas y de la documentación institucional

Han sido once las empresas entrevistadas, todas ellas incluidas en sectores creativos aunque realizando actividades muy diversas. Para su selección, se ha tenido en cuenta a las que, según información facilitada por las instituciones y organismos entrevistados, parecen destacar por su creatividad y capacidad innovadora, sin que ello en principio esté relacionado con el sector de actividad en el que operan, su forma jurídica o su tamaño.

Fundamentalmente, se trata de empresas pequeñas o microempresas, siendo relativamente joven la edad media de los empresarios. Estos últimos están en su mayoría directamente involucrados en la producción o en la prestación de servicios y muestran una creciente preocupación, tanto por la formación, como por la conciliación entre el carácter cultural del producto generado y la rentabilidad económica. Pese a la limitada internacionalización de estas empresas, se están desarrollando algunos proyectos de cooperación con empresas existentes en otros países, siendo, por el contrario, menos frecuentes los llevados a cabo entre firmas del ámbito local.

Han sido dos las editoriales entrevistadas: La Máquina China y El Mono Azul. La primera, cuya actividad se inicia en Sevilla en 2004, es una empresa especialmente creativa que empezó con un plan de negocios que se sustentaba en la puesta en valor del uso de Internet; para ello se asociaba a cada libro una página web en la que se incluía información sobre los personajes, el lugar y la época en que se desarrolla la trama, además de abrir foros en los que participaban los autores. Aunque esta empresa obtuvo varios premios, no resultó en principio muy rentable por lo que, partiendo de la experiencia adquirida, convirtió el fracaso en oportunidad, centrándose tanto en reajustar las tareas anteriores como en agilizar la gestión económica mediante la reducción de los tiempos, pero siempre teniendo en cuenta de forma destacada las reacciones de los lectores a través de internet. Ha publicado en papel y en formato digital y, además, realiza audio-guías; como complemento de lo anterior, ha impartido cursos en red y organizado visitas guiadas a ciudades y territorios en los que se desarrollan las tramas de los libros publicados. No obstante, como consecuencia de la crisis, ha tenido que suspender algunas de sus actividades en 2012.

El Mono Azul, por su parte, fue creada en 2005 en el municipio de Coria del Río y se dedica básicamente a la reedición de obras de calidad y a la promoción de autores intencio- 
nalmente seleccionados. Su competitividad se basa en su carácter independiente, a la vez que en aprovechar el valor creciente de la cooperación, lo que demuestra a través de su integración en la Red Internacional de Editores y Proyectos Alternativos (RIEPA).

Dentro del sector industrial, se incluye también la empresa Gloria Bendita, dedicada al diseño, producción y venta de ropa. Sus propietarios, teniendo en cuenta los problemas de gestión derivados del hecho de que la ropa que diseñaban en su pequeño taller de Ibiza se fabricaba en Barcelona, deciden trasladarse a Sevilla en 2005, ciudad en la que realizan tanto las tareas de diseño como las de fabricación. Empezaron contactando con el Proyecto Lunar, donde les asesoraron técnicamente además de facilitarle un módulo en su incubadora; allí permanecieron hasta 2010, año en el que abrieron su nueva sede. Junto al asesoramiento recibido a través del Proyecto Lunar, destaca también el obtenido a través del Centro Tecnológico del Textil, situado en Priego de Córdoba donde, además de asesorarles en la gestión, desarrollan sus prototipos. Trabajan bajo el principio de «Moda Ética» usando fibras naturales y cuidando socialmente sus formas de producción; en este contexto se incluyen los proyectos que actualmente desarrollan en Mali y en Gambia colaborando tanto con productores locales, como con la empresa Hermenegildo Zegna en la tutela de estudiantes africanos. Actualmente han cerrado la tienda, aunque siguen con la venta en internet.

Geographica Studio, que inicia su actividad en 2002, es una empresa de servicios avanzados relacionados con las tecnologías geoespaciales y geomédicas, las plataformas web y los juegos digitales. Entre las actividades realizadas cabe destacar, por ejemplo, el desarrollo de un juego para aprender japonés en colaboración con la empresa internacional Edge Entertainment; habiendo sido contratada por algunos organismos internacionales entre los que se encuentra la UNESCO. Geographica Studio fue Premio 2006 de la Universidad de Sevilla en la modalidad de Empresa Innovadora.

Dentro del sector de la fotografía, se ha entrevistado a la empresa Cobertura Photo, que se autodefine como un espacio de actividades relacionadas con la fotografía. Entre sus principales líneas de trabajo se incluyen la realización de proyectos, la organización de conferencias y proyecciones, así como la organización de cursos de formación que incluyen talleres magistrales impartidos por fotógrafos de reconocido prestigio. Junto a lo anterior, trabajan para incluir entre los servicio ofertados una librería especializada en fotografía. Hay que llamar la atención sobre el hecho de que, además de las actividades de carácter local, desarrollan proyectos ligados al Programa Cultura de la Unión Europea en tres de sus líneas: editorial, expositiva y pedagógica.

Entre las empresas dedicadas a ofrecer servicios ligados a la cultura se ha entrevistado a Engranajes culturales que, creada en 2009, vincula sus inicios a la participación de sus propietarios en un concurso convocado por la Universidad de Sevilla en el que resultaron ganadores; entre otras ventajas, el premio facilitaba la concesión de un espacio en la incubadora de empresas gestionada por CREA. Los servicios que ofertan son muy diversos, entre ellos destacan las visitas temáticas guiadas a museos e itinerarios urbanos centrados en temas concretos o dirigidos a determinados grupos sociales. Hay que mencionar también un proyecto desarrollado durante la Semana Santa de 2011 que, junto a las visitas a museos y los itinerarios urbanos asociados a este evento, incluye el acompañamiento personalizado a los desfiles procesionales. 
Se dedica también a la gestión cultural la empresa ACTIDEA que, creada en 2000, parece muy preocupada por introducir innovaciones relacionadas con la gestión, poniendo de manifiesto su preocupación por la mejora continua y la flexibilidad, características que considera básicas para mantener su competitividad. Aunque la empresa desarrolla sus actividades prioritariamente en el municipio de Sevilla, las lleva también a cabo en municipios de la corona metropolitana, en la provincia, en otras zonas de Andalucía y, en ocasiones puntuales, en Madrid. Una de sus actividades más conocidas es la organización de conciertos nocturnos en los jardines del Alcázar de Sevilla.

El Instituto Internacional de Estudios Técnicos y Emocionales, creado en 2009, realiza actividades que se centran fundamentalmente en la organización de cursos de formación, ya sean dirigidos específicamente a determinadas empresas o para ser compartidos por empresarios y directivos de diferentes organizaciones; todos ellos tienen como base la exploración y el aprovechamiento de los recursos emocionales para potenciar la creatividad de empresas e instituciones. Uno de estos cursos impartidos se integra en la oferta educativa de la Universidad de Sevilla. A su vez, desarrolla proyectos propios entre los que cabe destacar el conocido como Universidad Emocional, cuyo objetivo es investigar, experimentar y avanzar en el conocimiento de las emociones y sus posibles aplicaciones.

El Grupo TASO es una consultora creada en 1999 que trabaja fundamentalmente en el asesoramiento de estrategias de desarrollo económico y organización empresarial. En los últimos años, viene realizando trabajos de identificación y análisis de cluster creativos y espacios empresariales vinculados a este tipo de actividades.

Con un planteamiento diferente a los anteriormente analizados, en 2006 abre en Sevilla el Museo del Flamenco. Se trata de un proyecto museográfico privado y no convencional de carácter abierto en el que se integran también espectáculos, se imparten clases de baile y se editan libros.

Cabe citar, por último, a la empresa SVQ Investigación y Desarrollos Estratégicos que se encargó en su momento de desarrollar un proyecto titulado «Observatorio de Industrias Culturales de Andalucía», que fue financiado por el Ministerio de Cultura, aunque no ha desarrollado más que una aplicación web con cierta información sobre eventos y actividades culturales, que además no está actualizada.

Pese a la creatividad demostrada por muchas de estas empresas, al reducido tamaño de buena parte de ellas, a la limitada formación de algunos empresarios, a la escasa cultural de colaboración inter-empresarial y a las formas en que se gestionan, se suma ahora la fuerte incidencia que está teniendo en ellas la crisis, al reducirse el consumo de este tipo de productos y, sobre todo, al disminuir considerablemente los fondos públicos para su promoción y financiación, de los que dependen buena parte de las actividades que llevan a cabo estas empresas.

\section{RED DE COOPERACIÓN SOCIO-INSTITUCIONAL DE AGENTES CON INCIDENCIA EN LAS ACTIVIDADES CREATIVAS}

Son muchos los estudiosos interesados en el análisis de las redes locales de cooperación socio-institucional al entender que contribuyen a la creación de un entorno social que permite la búsqueda colectiva de soluciones a los problemas y disfunciones existentes en los distintos ámbitos territoriales. Dichas redes se convierten así en un paradigma asocia- 
tivo o paradigma de red que vincula la existencia de relaciones con el desarrollo territorial (Camagni, 1991; Cooke y Morgan, 1993; Casti, 1995; Koschatzky, 2002). Refiriéndose a la importancia de las redes, se manifiesta, por ejemplo, Subirats cuando plantea la hipótesis de que «aquellos territorios y comunidades que cuenten con esta base social y la aprovechen mejor, o sepan generar mecanismos para hacerla aflorar y para consolidarla, serán los mejor preparados para afrontar colectivamente su futuro» (Subirats, 2002: 18). Por su parte, Romero y Farinós (2011) señalan que no puede olvidarse que las redes de cooperación están estrechamente relacionadas con la gobernanza territorial en la que pueden destacarse dos dimensiones fundamentales: una vertical, debida a relaciones entre los diferentes niveles político-administrativos; y otra horizontal, que se conforma, a su vez, por tres tipos de relaciones: entre sectores, entre territorios y entre los distintos actores con presencia activa en el ámbito objeto de atención.

En relación con el contenido de este artículo, no hay que olvidar, por ejemplo, que entre los rasgos que han sido considerados básicos para definir la llamada ciudad creativa, Landry (2000) incluye a la cooperación. A su vez, y por lo que respecta a la economía creativa, «la antigua dicotomía entre lo público y lo privado ha sido colapsada [...] por una realidad compleja en la que conviven organizaciones, empresas e instituciones públicas junto a iniciativas sociales y económicas, como las fundaciones y asociaciones» dando paso «a interesadas e interesantes estrategias de cooperación entre los diversos actores y agentes, tengan estos o no intereses mercantiles» (Núñez de la Fuente, 2010: 203).

En España son ya numerosos los trabajos geográficos que, aplicando la metodología de análisis de redes, profundizan en el conocimiento del comportamiento socioeconómico de distintos tipos de territorios (distritos industriales, sistemas productivos locales agro-comerciales o turísticos...), poniendo en relación la presencia y la densidad de las redes con distintos niveles de desarrollo territorial (Alonso y Méndez, 2000; Caravaca, González; Méndez y Silva, 2002; Alonso, Aparicio y Sánchez, 2004; Salom y Albertos, 2009).

El estudio específico de los agentes en espacios concretos y de las redes locales de cooperación que, en su caso, éstos conforman, requiere de un sistemático trabajo de campo en el que se identifiquen los actores con presencia activa en el ámbito, los objetivos y estrategias que se plantean y las actuaciones que realizan. Una vez realizado este análisis, tal y como queda recogido en los apartados anteriores, se ha procedido a la identificación de los vínculos que conforman la red de cooperación que, asociada a las actividades creativas, se ha conformado en la aglomeración metropolitana de Sevilla.

Como puede observarse en la figura 3 , son poco numerosas las conexiones establecidas hasta ahora entre los diferentes actores con presencia activa en el ámbito, lo que explica el bajo grado de conectividad de la red, que únicamente alcanza el 21,32\% ${ }^{4}$. En efecto, sólo 10 de las 18 actuaciones de cooperación que han podido identificarse están dirigidas específicamente a actividades creativas; a ello hay que añadir que las relaciones son, por una parte, poco complejas, pues la mayoría de los vínculos existentes entre los agentes se sostienen sobre un sólo proyecto y, por otra, poco extensivas, al ser reducido el número de actores participantes.

4 Para cuantificar el grado de conectividad (GC) se relaciona el número de vínculos (a) con el número de agentes (n) que conforman la red a través de la fórmula siguiente: GC=2a/n(n-1). 
El Ayuntamiento de Sevilla ha ejercido hasta ahora un papel clave en esta red al haberse relacionado con un mayor número de agentes y participado en un mayor número de proyectos y actuaciones comunes. Por lo que se refiere a otros niveles administrativos, cabe llamar la atención sobre el autonómico, pues son varias las consejerías que participan activamente en proyectos colectivos; entre ellas desataca la de Economía, Innovación y Ciencia, no sólo por ser la más dinámica, sino porque es la que sirve de nexo de unión entre las principales organizaciones empresariales del sector audiovisual y el resto de los actores socio-institucionales que integran la red. Como contrapunto, la Diputación Provincial no parece estar colaborando con otros agentes. Aunque se establecen relaciones de cooperación entre el Ayuntamiento de Alcalá de Guadaíra con el Ministerio de Innovación y Ciencia, no llegan a integrarse en la red principal, conformando una subred.

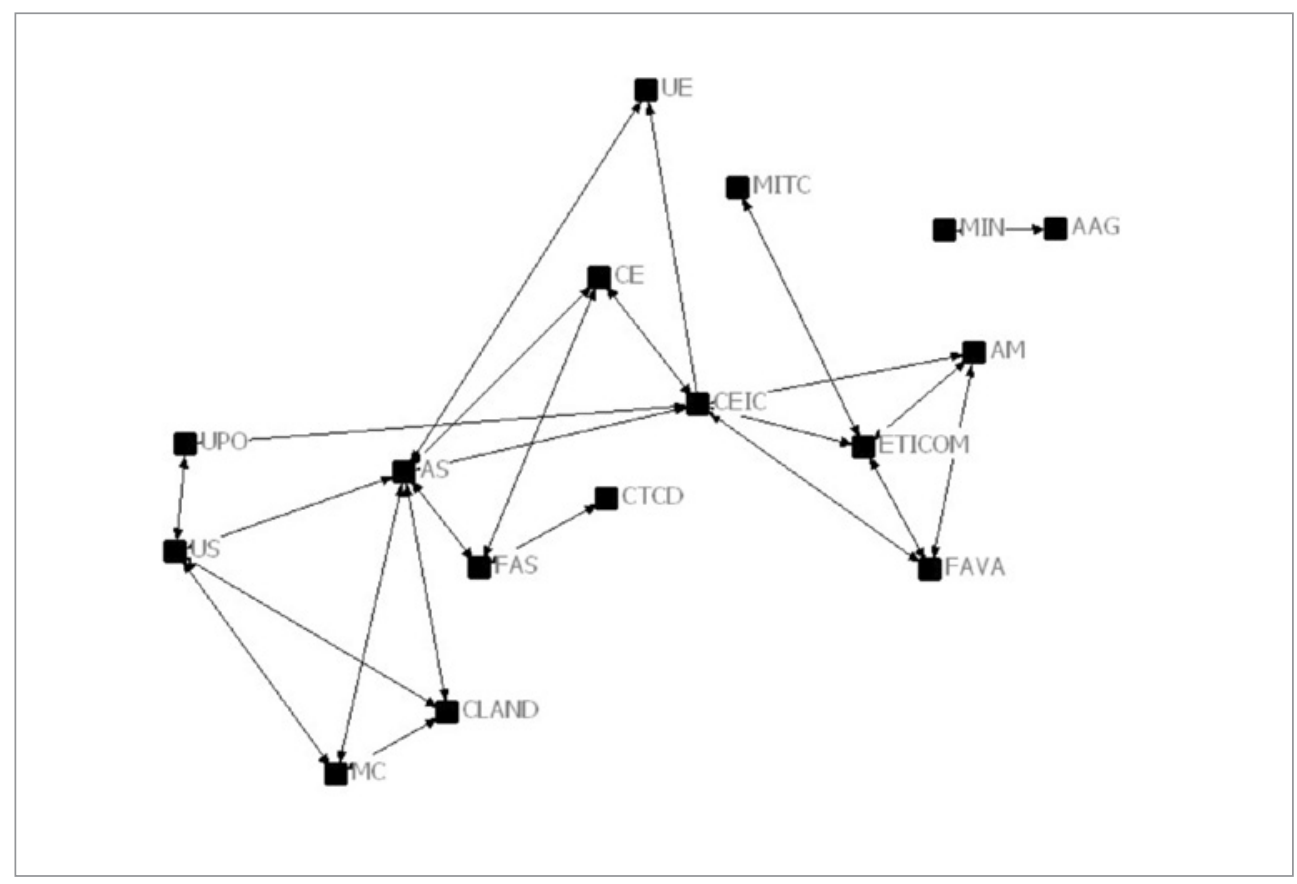

UE: Unión Europea; MC: Ministerio de Cultura; MIN: Ministerio de Innovación y Ciencia; MITC: Ministerio de Industria, Turismo y Comercio; CEIC: Consejería de Economía, Innovación y Ciencia; CE: Consejería de Empleo; CTCD: Consejería de Turismo, Comercio y Deporte; AS: Ayuntamiento de Sevilla; AAG: Ayuntamiento de Alcalá de Guadaíra; US: Universidad de Sevilla; UPO: Universidad Pablo de Olavide; FAVA: Fundación Audiovisual de Andalucía; CLAND: Asociación Cluster Digital y Audiovisual de Andalucía; AM: Asociación Masquelasuma de empresas audiovisuales y TIC; ETICOM: Asociación Empresarios TIC Andalucía; FAS: Federación de Artesanos de Sevilla.

Fuente: Elaboración propia a partir de las entrevistas y la documentación disponible 
Por su parte, son varias las asociaciones empresariales que están implicadas en proyectos colectivos; es el caso de la Federación de Artesanos de Sevilla y de las asociaciones del sector audiovisual. En relación con este último, hay que llamar la atención sobre la creación de un portal para favorecer la difusión de la información y la colaboración entre empresas de los sectores audiovisual y de tecnologías de la información y comunicación, así como la utilización conjunta de instalaciones como el Centro Demostrador TIC Andalucía y el Centro Cloud Computing. Esto explica los lazos establecidos entre la Fundación Audiovisual de Andalucía, patrocinada por la Agencia Pública Empresarial de la Radio y Televisión de Andalucía (RTVA), y otros actores del sector de las tecnologías de la información y comunicación.

El análisis de la red vuelve a evidenciar la ya mencionada falta de colaboración entre agentes públicos entre sí, y entre éstos y los privados, lo que redunda en una falta de gobernanza tan necesaria siempre para impulsar los procesos de desarrollo e imprescindible cuando, como ahora, se está inmerso en una profunda crisis.

\section{CONSIDERACIONES FINALES}

Las actividades creativo-culturales tienen un peso poco significativo en la estructura económica de la aglomeración metropolitana de Sevilla; pese a ello, este ámbito concentra buena parte de las existentes en Andalucía. En cuanto a su distribución sectorial, destacan tanto por el número de empresas como de empleos, las actividades de Arquitectura e ingeniería, Publicidad y estudios de mercado, Cine, televisión y música, y Programación y emisión de radio y TV; no obstante, se observan ciertas diferencias en el peso de las diferentes ramas entre la ciudad central y la corona metropolitana.

En relación con los agentes socio-institucionales del ámbito de estudio y el papel que éstos desempeñan en la promoción de las actividades creativo-culturales, las entrevistas en profundidad se han revelado como una fuente de información de suma importancia.

Aunque instituciones de todos los niveles administrativos realizan algún tipo de actuación vinculada a las actividades creativas, son las pertenecientes a la administración regional y al ayuntamiento de la ciudad central las que se muestran más activas. En el caso de la administración regional, hay que destacar el papel estratégico que ésta desarrolla no sólo como promotor sino como cliente clave para algunas actividades (artes escénicas, audiovisual y edición), lo que llega a generar conflictos entre algunas asociaciones empresariales.

A su vez, es interesante señalar cómo los departamentos de las administraciones que ejercen un papel más dinámico a la hora de emprender y formar parte de proyectos de dinamización o promoción de este tipo de actividades no pertenecen al ámbito de la cultura, sino que están más vinculados a la promoción económica.

Llama especialmente la atención que algunas estrategias y actuaciones lleguen a ser coincidentes y hasta se superpongan en ocasiones; ello demuestra un cierto desconocimiento de las mismas que puede ser debido a su escasa difusión, o bien, a un excesivo, además de innecesario, celo competencial. Se manifiesta, pues, que, pese al énfasis que se pone en la gobernanza en la mayor parte de documentos institucionales, ésta no llega a ser realmente asumida, haciéndose imprescindible un esfuerzo de coordinación inter e intra-institucional que permita un mejor aprovechamiento de los recursos y la generación de sinergias. 
En cuanto a las empresas, merece destacarse la creciente preocupación que muestran tanto por la formación como por conciliar el producto cultural con la rentabilidad económica. Aunque la colaboración empresarial es muy limitada, se están desarrollando algunos proyectos de cooperación internacionales y regionales, mientras que son menos frecuentes los llevados a cabo en el ámbito local.

Por lo que a la red de cooperación socio-institucional se refiere, se observa que son: poco extensivas, al ser reducido el número de actores que participan en la red; poco densas, al ser reducidos los vínculos entre actores; y poco complejas, dado que la mayoría de los vínculos existentes entre los agentes se sostienen sobre un único proyecto.

En definitiva, del análisis realizado puede concluirse que, además de ser limitada la presencia de las actividades creativas en el ámbito objeto de estudio, también es reducida su importancia económica. Junto a ello hay que considerar que una escasa capacidad de colaboración entre los agentes, tanto públicos como privados, dificulta aún más la capacidad de respuesta de este territorio frente a la grave crisis estructural que se viene padeciendo. Es evidente, pues, que continúa siendo un importante reto para esta aglomeración metropolitana potenciar este tipo de actividades -basadas en un recurso intangible y de difícil deslocalización al estar en buena parte asociado a la identidad cultural de este ámbito-, para que, aunque sea modestamente, puedan contribuir a la sustitución del actual modelo económico por otro nuevo, que a más de ser económicamente competitivo sea también y, sobre todo, ambientalmente sostenible, territorialmente equilibrado y socialmente cohesionado.

\section{BIBLIOGRAFÍA}

ALLENDE, J. (1995): «Desarrollo sostenible. De lo global a lo local». Ciudad y Territorio. Estudios Territoriales, $n^{\circ}$ 104, 287-292.

ALONSO, J.L. y MÉNDEZ, R. (Coords., 2000): Innovación, pequeña empresa y desarrollo local en España. Madrid, Civitas.

ALONSO, J.L; APARICIO, J. y SÁNCHEZ, J.L. Eds., (2004): Recursos territoriales y geografía de la innovación industrial en España. Salamanca, Ediciones Universidad de Salamanca.

BAYLISS, D. (2007) «The Rise of the Creative Citiy: Culture and Creativity in Copenhagen». European Planning Studies, no 15 (7), 889-903.

BOUQUILLION, P.; MIÈGE, B. y MOEGLIN, P. (2011): «La situación de la industria creativa. Un debate significativo en Francia»en BUSTAMANTE, E., Ed. Industrias creativas. Amenazas sobre la cultura digital Barcelona, Gedisa, 97-116.

BUSTAMANTE, E. Ed. (2011): Industrias creativas. Amenazas sobre la cultura digital. Barcelona, Gedisa.

CAMAGNI, R. Ed. (1991): Innovation networks. Spatial perspectives. Londres, GREMIBelhaven Press.

CARAVACA, I.; GONZÁLEZ G.; MÉNDEZ, R. y SILVA, R. (2002): Innovación y Territorio. Análisis comparado de sistemas productivos locales en Andalucía. Sevilla, Consejería de Consejería de Economía y Hacienda, Junta de Andalucía.

CARAVACA, I. y GONZÁLEZ, G. (2009): «Las redes de colaboración como base del desarrollo territorial», en Scripta Nova, vol XII, n 289, 1 de mayo de 2009. Universidad de Barcelona. Disponible en http://www.ub.es/geocrit/sn/sn-289.htm. 
CARAVACA, I.; GARCÍA, A. y GONZÁLEZ, G. (2011): «La aglomeración metropolitana de Sevilla en la sociedad del conocimiento», en Actas do XII Colóquio Ibérico de Geografia. Porto, Faculdade de Letras (Universidade do Porto).

CARAVACA, I.; GONZÁLEZ, G. y GARCÍA, A. (2011): «Nuevas tendencias socioeconómicas en los espacios urbanos. Las actividades creativas en la aglomeración metropolitana de Sevilla», en Urbanismo expansivo, de la utopía a la realidad (Gonzálvez, V. y Marco, J.A., eds.). Murcia, Asociación de Geógrafos Españoles y Universidad de Alicante. Tomo II, 103-118.

CASTI, J.L. (1995): «The Theory of Networks» en Networks in Action.Communication, Economics and Human Konowledge (Basten, D., J. Casti y R. Thord, eds.). Berlín, Springer, 3-24.

CAVES, R.E. (2000): Creative industries: Contracts between art and commerce. Harvard, Harvard University Press.

COMISIÓN EUROPEA (2007): Agenda Europea para la Cultura en un Mundo en vías de Globalización (COM, 2007, 242). Bruselas, Comisión Europea.

COMISIÓN EUROPEA (2009): Challenges for EU support to innovation services. Fostering new markets and jobs through innovation (SEC, 2009, 1195). Bruselas, Comisión Europea.

COMISION EUROPEA (2010 a): Libro Verde. Liberar el potencial de las industrias culturales y creativas. Bruselas, Comisión Europea.

COMISION EUROPEA (2010 b): Europa 2020: Una estrategia para un crecimiento inteligente, sostenible e integrador. Bruselas, Comisión Europea.

COMISIÓN EUROPEA (2010 c): Contribución de la Política Regional al crecimiento inteligente en el marco de Europa 2020 SEC(2010) 1183. Comunicación de la Comisión al Parlamento Europeo, al Consejo, al Comité Económico y Social Europeo y al Comité de las Regiones. Bruselas, Comisión Europea.

CONSEJERÍA DE EMPLEO (2011): Caracterización general de la industria cultural en Andalucía. Sevilla, Junta de Andalucía. Disponible en http://www.empleocultural.es.

COOKE, P. y MORGAN, K. (1993): «The Network Paradigm. New Departures In. Corporate and Regional Development». Society and Space, $\mathrm{n}^{\circ}$ 11, 543-564.

COOKE, P. y LAZZERETTI, P. (2008): Creative cities, cultural clusters and local economic development. Cheltenham-Northamton, Edwaerd Elgar.

COSTA, P. (2008): «Creativity, Innovation and Territorial Agglomeration in Cultural Activities: The Roots of the Creative City» en Creative Cities, Cultural Clusters and Local Economic Development (Cooke, P. y Lazzeretti, L., eds.). Cheltenham-Northamton, Edwaerd Elgar, 183-210.

DEPARTMENT OF CULTURE, MEDIA AND SPORTS (DCMS) (1998): Creative Industries Mapping Document. Londres, Department for Culture, Media and Sport.

DEPARTMENT OF CULTURE, MEDIA AND SPORTS (DCMS) (1999): Creative Industries Export: Our Hidden Potential. Londres, Department for Culture, Media and Sport.

EVANS, G. (2009): «Creative Cities, Creative Spaces and Urban Policy». Urban Studies, ${ }^{\circ}$ 46 (5\&6), 1003-1040.

FLORIDA, R. (2009): Las ciudades creativas. Barcelona, Paidós.

FOORD, J. (2009): «Strategies for Creative Industries: An International Review». Creative Industries Journal, $\mathrm{n}^{\mathrm{o}} 1$ (2), 91-113. 
GIBSON, C. y KONG, L. (2005): «Cultural Economy: A Critical Review». Progress in Human Geography, no 29 (5), 541-561.

GALLOWAY, S. y DUNLOP, S. (2007): «A critique of definitions of the cultural and creative industries in public policy». International Journal of Cultural Policy, $\mathrm{n}^{\mathrm{o}} 13$, 17-31.

GARCÍA GARCÍA, A., FERNÁNDEZ SALINAS, V., CARAVACA BARROSO, I. y GONZÁLEZ ROMERO, G. (2012): «Cultural resources and creative industries in spanish medium-sized cities». Brazilian Geographical Journal: Geosciences and Humanities research medium, Uberlândia, v. 3, nº 1, 50-69

GARNHAN, N. (2005): «From cultural to creative industries». International Journal of Cultural Policy, $\mathrm{n}^{\circ} 11,15-29$.

GARNHAN, N. (2011): «De las industrias culturales a las creativas. Análisis de las implicaciones en el Reino Unido», en Industrias creativas. Amenazas sobre la cultura digital (Bustamante, E. ed.) Barcelona, Gedisa Ed, 21-48.

HOSPERS, G.J. (2003): «Creative Cities. Breeding Places on the Knowledge Economy». Knowledge, Technology and Policy, $\mathrm{n}^{\circ} 16$ (3), 143-172.

JUNTA DE ANDALUCÍA (2006): Plan de Innovación y Modernización de Andalucía. Sevilla, Consejería de Innovación, Ciencia y Empresa.

KEA EUROPEAN AFFAIRS (2006): The economy of culture in Europe. Bruselas, European Commisión Directorate General for Education and Culture. Disponible en http://www. keanet.eu/ecoculture/executive_summary_en.pdf

KEA EUROPEAN AFFAIRS (2009): The impact of culture on creativity. Bruselas, European Commisión Directorate General for Education and Culture. Disponible en http://www. keanet.eu/docs/impactculturecreativityfull.pdf

KOSCHATZKY, K. (2002): «Fundamentos de la Economía de Redes. Especial enfoque a la innovación». Economía Industrial, 2002, no 346, 15-26.

LANDRY, C. (2000): The Creative City: A Toolkit for Urban Innovators. London: Earthscan. LESLIE, D. (2005): «Creative cities?». Geoforum, no 36, 403-405.

MANITO, F. Ed. (2009): Ciudades Creativas. Volumen 1. Cultura, territorio, economía y ciudad. Barcelona, Fundación KREANTA.

MANITO, F. Ed. (2010): Ciudades Creativas. Volumen 2. Creatividad, innovación, cultura y agenda local. Barcelona, Fundación KREANTA.

MANITO, F. Ed. (2011): Ciudades Creativas. Volumen 3. Economía creativa, desarrollo urbano y políticas públicas. Barcelona, Fundación KREANTA.

MARCEL, X. (2009): «Ciudades creativas: ciudad, talento, innovación. 10 notas a pie de página» en Ciudades Creativas. Volumen 1. Cultura, territorio, economía y ciudad (Manito, F., ed.). Barcelona, Fundación KREANTA, 139-144.

MAS SERRA, E. (2009): «Ciudad, identidad y ranking». EURE, vol. XXXV, n 106, 29-49.

MÉNDEZ, R., MICHELIN, J., PRADA, J. y TÉBAR, J. (2012): «Economía creativa y desarrollo urbano en España: Una aproximación a sus lógicas espaciales». EURE, Revista Latinoamericana de Estudios Urbanos y Regionales, $\mathrm{n}^{\circ}$ 113, 5-32.

NUÑEZ DE LA FUENTE, J.M. (2010): «La colaboración entre instituciones, organizaciones y empresas culturales» en Economía y empleo en la cultura (Hernández Pavón, E., coord.). Sevilla, Junta de Andalucía. Consejería de Cultura, 203-212. 
O'CONNOR, J. (2008): The cultural and creative industries: a review of the literature. A report for Creative Partnerships. Londres, Arts Council of England.

ORTEGA VALCÁRCEL, J. (1998): «El patrimonio territorial como recurso cultural y económico, Ciudades». Revista del Instituto de Urbanística de la Universidad de Valladolid, n 4, 33- 48.

PRATT, A. (2008): «Creative Cities?». Urban Design Journal, nº 106, 35-60.

RALLET, A. (1995): «Ressources spécifiques et ressorces g'enériques: une problématique pour le développment local» en Les nouvelles logiques du développement (Abdelmaki, L. y Courlet, C., eds). París, L'Harmattan, 39- 57.

ROMERO, J. y FARINOS, X. (2011): «Redescubriendo la gobernanza más allá del buen gobierno. Democracia como resultado». Boletín de la AGE, $\mathrm{n}^{\circ}$ 56, 295-319.

SALOM, J. ALBERTOS, J.M. Eds. (2009): Redes socioinstitucionales, estrategias de innovación y desarrollo territorial en España. Valencia, Universidad de Valencia.

SÁNCHEZ MORAL, S. (2011): «Contribución de los servicios al desarrollo urbano en el contexto de la globalización y la sociedad del conocimiento» en HERNÁNDEZ LUÍS, J.A. Ed. Servicios, Globalización y Territorio. Madrid-Las Palmas de Gran Canaria, Anroart Ediciones. Colección Textos Universitarios, Tomo I, 211-258.

SÁNCHEZ ZAPATA, P. y ÁLVARO JULIO, F. (2009): «Apoyo integral a emprendedores creativos en Sevilla» en Ciudades Creativas. Volumen 1 Cultura, territorio, economía y ciudad, (Manito, F., ed.). Barcelona, Fundación KREANTA, 101-109.

SCOTT, A.J. (2006): «Creative cities: Conceptual issues and policy questions». Journal of Urban Affairs, 28, Vol. 1, 1-17.

SCHLESINGER, P. (2011): «Intelectuales y políticas culturales. La política británica de industrias creativas» en Industrias creativas. Amenazas sobre la cultura digital en Bustamante, E., Ed. Barcelona, Gedisa, 81-96.

SUBIRATS, J. Coord. (2002): Redes, territorios y gobierno. Nuevas respuestas locales a los retos de la globalización. Barcelona, Universidad Internacional Menéndez Pelayo.

TREMBLAY, G. (2011): «Desde la teoría de las industrias culturales. Evaluación crítica de la economía de la creatividad» en Bustamante, E., Ed, Industrias creativas. Amenazas sobre la cultura digital, Barcelona, Gedisa, 49-80.

THROSBY, D. (2001): Economía y cultura. Madrid, Cambridge University Press

UNCTAD (2004): Creative Industries and Development. Washington DC, United Nations Conference on Trade and Development, UNCTAD.

UNCTAD (2008): Creative Economy Report 2008. The Challenge of Assessing the Creative Economy towards informed policy making, Washington DC, United Nations Conference on Trade Development, UNCTAD. Disponible en http://www.unctad.org/creative-economy

UNESCO (2006): Understanding creative industries: cultural statistics for public-policy making. Paris, UNESCO.

UNESCO (2009): The 2009 UNESCO Framework for Cultural Statistics (FCS). Montreal (Canada), Institute for Statistics of the United Nations Educational Scientific and Cultural Organization (UIS).

UNIÓN EUROPEA (2009): Manifiesto de los Embajadores del Año Europeo de la Creatividad y la Innovación. Bruselas, Comisión Europea. Disponible en http://www.create2009. europa.eu/ambassadors.html. 
